\title{
LETTERS
}

The purpose of this Letters section is to provide rapid dissemination of important new results in the fields regularly covered by Physics of Fluids A. Results of extended research should not be presented as a series of letters in place of comprehensive articles. Letters cannot exceed three printed pages in length, including space allowed for title, figures, tables, references and an abstract limited to about 100 words. There is a three-month time limit, from date of receipt to acceptance, for processing Letter manuscripts. Authors must also submit a brief statement justifying rapid publication in the Letters section.

\section{Some consequences of the boundedness of scalar fluctuations}

\author{
Paul E. Dimotakis and Paul L. Miller \\ Graduate Aeronautical Laboratories, California Institute of Technology, Pasadena, California 91125
}

(Received 24 August 1990; accepted 11 September 1990)

\begin{abstract}
Values of the scalar field $c(\mathbf{x}, t)$, if initially bounded, will always be bounded by the limits set by the initial conditions. This observation permits the maximum variance $\overline{c^{\prime 2}}$ to be computed as a function of the mean value $\bar{c}$. It is argued that this maximum should be expected in the limit of infinite Schmidt numbers (zero scalar species diffusivity). This suggests that $c^{\prime} / \bar{c}$ on the axis of turbulent jets, for example, may not tend to a constant, i.e., independent of $x / d$, in the limit of very large Schmidt numbers. It also underscores a difficulty with the $k^{-1}$ scalar spectrum proposed by Batchelor [J. Fluid Mech. 5, 113 (1959) ].
\end{abstract}

In contrast to velocity fluctuations in a three-dimensional flow, a turbulent flow cannot generate passive scalar fluctuaton values outside the initial bounds. Consequently, the values of the scalar field $c(\mathbf{x}, t)$, if initially within 0 and 1 , for example, will always be bounded by these limits. This can be shown to be a direct result of the scalar transport equation,

$$
\frac{\partial c}{\partial t}+\mathbf{u} \cdot \nabla c=\mathscr{D} \nabla^{2} c
$$

in which the scalar species diffusivity $\mathscr{D}$ must be positive, as directed by thermodynamic considerations.

A useful measure of the scalar fluctuations is the scalar variance $\overline{c^{\prime 2}}$, which equals the second moment of $p(c)$, the probability density function (pdf) of the scalar, i.e.,

$$
\overline{c^{\prime 2}}=\int_{0}^{1}(c-\bar{c})^{2} p(c) d c,
$$

where the scalar values specified by the initial/boundary conditions have been scaled such that $0 \leqslant c \leqslant 1$ and where

$$
\bar{c}=\int_{0}^{1} c p(c) d c
$$

is the mean (expectation) value of the scalar field (pdf).

As a consequence of the boundedness of the values of the scalar field, the variance is maximized when the pdf realizes its integral as far away from the mean as possible. In other words, as

$$
p(c) \rightarrow(1-\bar{c}) \delta(c)+\bar{c} \delta(1-c),
$$

where $\delta(c)$ denotes the Dirac delta function. This pdf should describe the behavior in the limit of $\mathscr{D} \rightarrow 0$, corresponding to $\mathrm{Sc} \equiv v / \mathscr{D} \rightarrow \infty$, where Sc is the Schmidt number of the diffusing species and $v$ is the kinematic viscosity. In such a flow, the turbulence will be stirring, but not mixing, $\bar{c}$ parts of $c=1$ fluid per $(1-\bar{c})$ parts of $c=0$ fluid. This yields

$$
\overline{c^{\prime 2}} \rightarrow \bar{c}(1-\bar{c}) \quad \text { as } \mathrm{Sc} \rightarrow \infty
$$

for the limiting value for the variance, which attains its maximum of $\overline{c^{\prime 2}}=\frac{1}{4}$ at $\bar{c}=\frac{1}{2}$. The foregoing suggests that the ratio $\Xi$,

$$
0 \leqslant \Xi=\frac{\overline{c^{\prime 2}}}{\bar{c}(1-\bar{c})} \leqslant 1,
$$

is an appropriate unmixedness parameter, with zero attained in the limit of perfect mixing, i.e., as $p(c) \rightarrow \delta(c-\bar{c})$, and unity corresponding to stirring but no mixing [Eqs. (4) and (5)] in the limit of $S c \rightarrow \infty$. The quantity $\Xi$ of Eq. (6) was proposed by Danckwerts, ${ }^{1}$ in the context of estimating the rate of a chemical reaction of imperfectly mixed reactants, who dubbed it "intensity of segregation" and recognized its limits. In contrast, the more conventional normalization of the variance of the scalar fluctuations yields

$$
\overline{c^{\prime 2}} / \bar{c}^{2} \rightarrow(1-\bar{c}) / \bar{c} \text { as } \mathrm{Sc} \rightarrow \infty,
$$

and can realize any value between zero and infinity depending on the value of $\bar{c}$.

These relations imply that, in the limit of $\mathrm{Sc} \rightarrow \infty$, the normalized root mean square scalar fluctuations on the axis of a momentum-driven, axisymmetric, turbulent jet, for example, should tend to infinity as $x / d \rightarrow \infty$, where $d$ is the jet nozzle diameter and $x$ is the distance from the jet nozzle (measured from a virtual origin). In particular, at finite Reynolds number and large $x / d$ we should expect

$$
\frac{c^{\prime}}{\bar{c}} \leqslant\left(\frac{1-\bar{c}}{\bar{c}}\right)^{1 / 2} \sim \text { const }\left(\frac{x}{d}\right)^{1 / 2} \text { as } \mathrm{Sc} \rightarrow \infty,
$$

since $\bar{c} \propto d / x$ in this case. This should be compared to the assumption that, at large $x / d, c^{\prime} / \bar{c}$ can be taken as a constant on the jet axis; often accepted as valid independently of the Schmidt number.

A second consequence of the limiting behavior of $\overline{c^{\prime 2}}$ concerns the spectrum of the scalar fluctuations at high Schmidt numbers. Specifically, it was proposed by Batche$\operatorname{lor}^{2}$ that at high Reynolds numbers and for spatial scales 
below the Kolmogorov dissipation scale ${ }^{3}$

$$
\lambda_{\mathrm{K}} \equiv\left(v^{3} / \epsilon\right)^{1 / 4},
$$

where $\epsilon$ denotes the dissipation rate of the kinetic energy per unit mass, the spectrum $E_{c}(k)$ of a passive conserved scalar $c(\mathbf{x}, t)$ should obey a $k^{-1}$ power law. This regime was predicted to extend to spatial scales down to the scalar diffusion (Batchelor) scale

$$
\lambda_{\mathrm{B}} \equiv \lambda_{\mathrm{K}} \mathrm{Sc}^{-1 / 2} \text {. }
$$

It would appear that the arguments leading to this conclusion become more robust as the separation between the Kolmogorov and Batchelor scales increases, i.e., in the limit of large Schmidt numbers.

Now, the variance of the scalar fluctuations can also be computed as the integral of the scalar spectrum over the range of wave numbers, i.e.,

$$
\overline{c^{\prime 2}}=\frac{1}{\pi} \int_{0}^{\infty} E_{\mathrm{c}}(k) d k \approx \frac{1}{\pi} \int_{0}^{1 / \lambda_{\mathrm{B}}} E_{\mathrm{c}}(k) d k .
$$

As a consequence, as Sc becomes large, the Batchelor proposal yields at high Reynolds numbers (Moffatt ${ }^{4}$ )

$$
\overline{c^{\prime 2}} \sim \frac{\chi}{U / L}\left(A+\frac{B}{2} \mathrm{Re}^{-1 / 2} \ln (\mathrm{Sc})\right),
$$

where

$$
\chi \equiv \overline{\mathscr{D}|\nabla c|^{2}} \sim \frac{(\Delta c)^{2}}{L / U}
$$

is the scalar dissipation rate, $\operatorname{Re}=L U / v$ is the flow Reynolds number, $A$ and $B$ are constants, and $\Delta c=1$ is the scalar diference specified by the initial/boundary conditions. This estimate of the variance can be seen to diverge (logarithmically) as the Schmidt number is increased.

The difficulty with the logarithmic divergence was recognized by Batchelor, ${ }^{2}$ who stated that the $k^{-1}$ spectrum would be established throughout the viscous-convective range, $1 / \lambda_{\mathrm{K}}<k<1 / \lambda_{\mathrm{B}}$, only if sufficient variance $\overline{c^{\prime 2}}$ was supplied from the larger scales (also see the discussion in Monin and Yaglom, ${ }^{5}$ p. 437). Moffatt ${ }^{4}$ points to the logarithmic divergence, but observes that, at high Reynolds numbers, the contribution from the Batchelor spectrum [ the second term in Eq. (11a)] is typically negligible.

We appreciate that to establish the logarithmic divergence of Eq. (11) may require a time $t$ in excess of the Batchelor diffusion time $t_{\mathrm{B}}$, which, for high Reynolds number flow, would be scaled by ${ }^{2}$

$$
t_{\mathrm{B}}=\left(t_{\mathrm{K}} / 2\right) \ln (\mathrm{Sc}),
$$

where

$$
t_{\mathrm{K}} \equiv(v / \epsilon)^{1 / 2} \propto(L / U) \mathrm{Re}^{-1 / 2}
$$

is the Kolmogorov dissipation time. ${ }^{3} \mathrm{~A}$ time $t_{\mathrm{B}}$ may be required for the diffusion interface thickness to decrease to the Batchelor diffusion scale $\lambda_{B}$, under the action of the subKolmogorov scale strain rate, and for the spectrum to extend to $k \approx 1 / \lambda_{\mathrm{B}}$. At fixed Reynolds number, the limiting behavior can be studied by increasing the Schmidt number, with an attendant increase in the time $t$ allowed for the diffusion process to attain dynamic equilibrium, such that $t>t_{\mathrm{B}}$, as necessary.

Comparing Eqs. (11) and (12), we see that the contribution to the variance from the $k^{-1}$ portion of the scalar spectrum is proportional to the product $\chi t_{\mathrm{B}} \sim \mathrm{Re}^{-1 / 2} \ln (\sqrt{\mathrm{Sc}})$. It would appear that, provided the time available for diffusion is larger than $t_{\mathrm{B}}$ as $\mathrm{Sc} \rightarrow \infty$, the $k^{-1}$ spectrum yields a scalar fluctuation variance that is inconsistent with the upper bound of Eq. (5).

Finally, we should note that a correction to the $k^{-1}$ power law that would increase the negative exponent of $k$, or the application of an integrating factor in that regime, would permit the integral over wave numbers to converge in the limit of high Schmidt number. We also recognize, however, that convergence alone would not be sufficient to resolve the difficulty; the scalar variance, as estimated by the scalar spectrum [Eq. (10)], must be bounded by its maximum [Eq. (5)].

\section{ACKNOWLEDGMENTS}

We would like to acknowledge our discussions with $P$. Saffman, D. Meiron, and H. K. Moffatt.

This work is part of a larger effort on turbulent mixing supported by AFOSR Grant No. 88-0155 and GRI Contract No. 5087-260-1467.

'P. V. Danckwerts, Appl. Sci. Res. Sec. A 3, 279 (1952).

${ }^{2}$ G. K. Batchelor, J. Fluid Mech. 5, 113 (1959).

${ }^{3}$ A. N. Kolmogorov, Dokl. Akad. Nauk SSSR 30, 299 (1941).

${ }^{4}$ H. K. Moffat, Rep. Prog. Phys. 46, 621 (1983).

${ }^{5}$ A. S. Monin and A. M. Yaglom, Statistical Fluid Mechanics: Mechanics of Turbulence, edited by J. Lumley (MIT Press, Cambridge, MA, 1975), Vol. II. 